The University of San Francisco

USF Scholarship: a digital repository @ Gleeson Library |

Geschke Center

1998

\title{
Interactions of Topological Kinks in Two Coupled Rings of Nonlinear Oscillators
}

\author{
A E. Duwel \\ C P. Heij \\ J C. Weisenfeld \\ Stephen M.K. Yeung \\ University of San Francisco, yeung@math.usfca.edu \\ E Trias
}

See next page for additional authors

Follow this and additional works at: http://repository.usfca.edu/math

Part of the Geometry and Topology Commons

\section{Recommended Citation}

A.E. Duwel, C.P. Heij, J.C. Weisenfeld, M.K.S. Yeung, E. Trias, S.J.K Vardy, H.S.J. van der Zant, S.H. Strogatz, and T.P. Orlando. Interactions of topological kinks in two coupled rings of nonlinear oscillators. Physical Review B 58, 8749-8754 (1998). Copyright American Physical Society. 
Authors

A E. Duwel, C P. Heij, J C. Weisenfeld, Stephen M.K. Yeung, E Trias, S. J.K. Vardy, H. S.J. van der Zant, S H. Strogatz, and T P. Orlando 


\title{
Interactions of topological kinks in two coupled rings of nonlinear oscillators
}

\author{
A. E. Duwel \\ Department of Electrical Engineering \& Computer Science, Massachusetts Institute of Technology, Cambridge, Massachusetts 02143 \\ C. P. Heij \\ Department of Applied Physics and DIMES, Delft University of Technology, P. O. Box 5046, 2628 CJ Delft, The Netherlands \\ J. C. Weisenfeld and M. K. Stephen Yeung \\ Department of Theoretical \& Applied Mechanics, Cornell University, Kimball Hall, Ithaca, New York 14853 \\ E. Trías \\ Department of Electrical Engineering \& Computer Science, Massachusetts Institute of Technology, Cambridge, Massachusetts 02143 \\ S. J. K. Várdy and H. S. J. van der Zant \\ Department of Applied Physics and DIMES, Delft University of Technology, P. O. Box 5046, 2628 CJ Delft, The Netherlands \\ S. H. Strogatz \\ Department of Theoretical \& Applied Mechanics, Cornell University, Kimball Hall, Ithaca, New York 14853 \\ T. P. Orlando \\ Department of Electrical Engineering \& Computer Science, Massachusetts Institute of Technology, Cambridge, Massachusetts 02143
}

(Received 27 April 1998; revised manuscript received 19 June 1998)

\begin{abstract}
Two discrete rings of nonlinear oscillators with topologically trapped kinks exhibit features due to coupling interactions between the rings. These interaction effects include phase locking between kinks in different rings, precession of the kink/antikink collision region, excitation of kink/antikink pairs, and time-dependent switching. We study these phenomena in simulations of two coupled discrete sine-Gordon equations, and in experiments on two inductively coupled rings of niobium Josephson junctions. [S0163-1829(98)06237-7]
\end{abstract}

\section{INTRODUCTION}

Discrete rings of coupled nonlinear oscillators have long served as model systems in studies of spatiotemporal pattern formation. Turing's pioneering analysis of morphogenesis ${ }^{1}$ was largely concerned with instabilities and spatial patterns in a discrete ring of $N$ cells, each governed by nonlinear chemical kinetics and coupled together by diffusion. Rings of nonlinear oscillators have also been used to model arrays of physical systems composed of phase-locked loops, ${ }^{2-4}$ lasers, ${ }^{5}$ Josephson junctions, ${ }^{6,7}$ pendula, ${ }^{8}$ chemical oscillators, ${ }^{9}$ and chaotic circuits. ${ }^{10}$

Certain kinds of rings have an interesting topological property: they can trap an integer number of kinks. To be concrete, consider an open-ended chain of pendula coupled to their nearest neighbors by torsional springs. Twist the chain a few times and then form a ring by connecting the first and last pendula by another torsional spring. Assuming that the springs remain intact, the net number of twists always remains constant, no matter how the system evolves. Winfree $^{11}$ calls this principle "the conservation of winding number.' It holds whether the twists spread out or form localized kinks and antikinks. More generally, trapped kinks can occur in any ring of elements satisfying the following conditions: the state of each element involves a circular phase variable, and the state's amplitude does not vanish anywhere along the ring. Trapped kinks have been studied in model rings of biological and chemical oscillators with strongly attracting limit cycles, ${ }^{11,12}$ and in long annular Josephson junctions. ${ }^{13-17}$

The dynamics are particularly rich if the oscillators are underdamped and the ring is discrete. Then, for small forcing, the twists remain localized. As these kinks propagate, they excite small-amplitude linear waves in their wake. ${ }^{18,19}$ When driven at certain speeds, a rotating kink can phase lock with its own radiation, leading to novel resonances that have recently been predicted ${ }^{6}$ and observed experimentally in discrete Josephson rings. ${ }^{20}$ Because of the discreteness, it is also possible for kinks and antikinks to travel at different speeds in a single system, giving rise to quasiperiodic resonances with more complicated spatiotemporal patterns. ${ }^{7,21}$

In this article, we explore a system of two discrete rings of underdamped oscillators, using inductively coupled Josephson junctions as an experimental realization. The nonlinear dynamics of two coupled discrete rings are almost uncharted. However the continuous counterpart, a stacked long Josephson junction ring system, has been studied experimentally, ${ }^{22,23}$ numerically, ${ }^{24}$ and analytically. ${ }^{25}$ An interesting feature is the phase locking between kinks and antikinks in the two rings. Such a phase-locking has been observed in continuous ring-systems. ${ }^{22,28}$ Together with the literature describing symmetries shared by (open-ended) continuous and discrete coupled systems, ${ }^{26,27}$ we approach the discrete coupled ring system with a certain intuition as to 


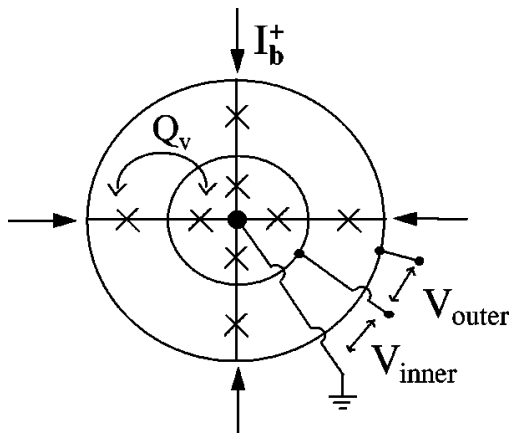

FIG. 1. Schematic of inductively coupled ring system. A uniform current $I_{\mathrm{b}}$ is fed into each node, as indicated by arrows and is extracted from the center island. In experiments, we measure the dc voltages $V_{\text {inner }}$ and $V_{\text {outer }} . Q_{\mathrm{v}}$ is the mutual inductance between two adjacent cells of the inner and outer rings.

the types of phenomena which might be observed.

Besides the phenomena seen in single discrete rings (trapping of kinks, resonances between kinks and linear waves in the same ring), we report fascinating effects that occur because of interactions between the rings. Our results include phase-locking between kinks in separate rings, a precession of the kink/antikink collision region in the ring, and an extreme sensitivity of excited states to the system parameters. We view this Josephson array as a model system for studying spatiotemporal dynamics of coupled oscillators, and more specifically for exploring kink interactions in discrete lattices, a topic that is also important in, e.g., the dynamics of dislocations and ferromagnetic domain walls. ${ }^{29,30}$

\section{THE SYSTEM}

The layout of a discrete coupled ring system with $N$ junctions per ring is shown in Fig. 1 for $N=4$. Current is fed into the nodes of the outer ring (shown by arrows), flows radially through the junctions and is extracted at the center island. The $\mathrm{Nb}-\mathrm{AlO}_{\mathrm{x}}-\mathrm{Nb}$ junctions are $3 \times 3 \mu \mathrm{m}^{2}$, and the critical current densities of the samples measured are in the range of $100 \mathrm{~A} / \mathrm{cm}^{2}{ }^{31}$ The radius of the outer ring is $28 \mu \mathrm{m}$. When the system is cooled in the presence of a perpendicular magnetic field to below the superconducting transition, the total flux bounded by the continuous superconducting rings becomes trapped in units of $\Phi_{o}=h / 2 e$. A single unit of quantized flux is called a vortex if the flux is along the applied field and an antivortex if it is opposite. A vortex and antivortex correspond to a kink and antikink. If there are $m_{\mathrm{v}, \text { in }}$ and $m_{\mathrm{v}, \text { out }}$ kinks in the two rings, and $m_{\mathrm{a} \text {,in }}$ and $m_{\mathrm{a} \text {,out }}$ antikinks, then the net, conserved phase winding in each ring is $M_{\text {in }}$ $=m_{\mathrm{v}, \text { in }}-m_{\mathrm{a}, \text { in }}$ and $M_{\mathrm{out}}=m_{\mathrm{v}, \text { out }}-m_{\mathrm{a} \text {,out }}$.

We use $\phi_{j}$ and $\psi_{j}$ to represent the phase of junction $j$ in the outer ring and inner ring, respectively. The dynamics are governed by two coupled discrete sine-Gordon equations:

$$
\begin{aligned}
\mathcal{N}\left[\phi_{j}\right]-\Lambda_{\text {out }}^{2} \nabla^{2} \phi_{j}-I_{b} / I_{c}= & Q_{\mathrm{v}, \text { out }} \mathcal{N}\left[\psi_{j}\right]+Q_{\mathrm{h}, \text { out }}\left(\mathcal{N}\left[\phi_{j+1}\right]\right. \\
& \left.+\mathcal{N}\left[\phi_{j-1}\right]\right), \\
\mathcal{N}\left[\psi_{j}\right]-\Lambda_{\mathrm{in}}^{2} \nabla^{2} \psi_{j}-I_{b} / I_{c}= & Q_{\mathrm{v}, \text { in }} \mathcal{N}\left[\phi_{j}\right]+Q_{\mathrm{h}, \mathrm{in}}\left(\mathcal{N}\left[\psi_{j+1}\right]\right. \\
& \left.+\mathcal{N}\left[\psi_{j-1}\right]\right),
\end{aligned}
$$

where $\mathcal{N}[\varphi(t)] \equiv \ddot{\varphi}+\Gamma \dot{\varphi}+\sin \varphi$ returns the total normalized current through a junction in response to its phase $\varphi(t)$, and $\nabla^{2} \varphi_{j}=\varphi_{j+1}-2 \varphi_{j}+\varphi_{j-1}$ is the discrete Laplacian. The bias current is normalized to the Josephson supercurrent, $I_{c} . \Gamma$ measures the damping in the system. In our normalization, the Josephson relation gives the instantaneous voltage at a junction as: $\dot{\varphi}_{j} \Gamma=V_{j} / I_{\mathrm{c}} R_{\mathrm{n}}$, where $R_{\mathrm{n}}$ is the junction's normal-state resistance. Experimentally, we measure the dc voltage across a ring, which is proportional to the spatiotemporally averaged $\langle\dot{\varphi}\rangle$. The parameters $\Lambda_{\text {in }}^{2}=L_{J} / L_{\text {in }}$ and $\Lambda_{\text {out }}^{2}=L_{J} / L_{\text {out }}$ are the ratios of the Josephson inductance $L_{J}$ $=\Phi_{o} / 2 \pi I_{c}$ to the cell inductances, $L_{\text {in }}$ and $L_{\text {out }}$. (Due to the planar geometry, we have $L_{\text {in }}<L_{\text {out }}$.) These ratios measure the spatial extent of localized kinks in each ring. The magnetic coupling between the two rings is dominated by the mutual inductance of adjacent cells in each ring, $Q_{\mathrm{v}}$ (see Fig. 1). The nearest-neighbor coupling within a ring $Q_{\mathrm{h}}$ is also included. All inductances are normalized to the selfinductance of a cell, giving $Q_{\mathrm{v}, \mathrm{x}}=Q_{\mathrm{v}} / L_{\mathrm{x}}$ and $Q_{\mathrm{h}, \mathrm{x}}=Q_{\mathrm{h}} / L_{\mathrm{x}}$, where " $x$ " refers to either "in' or "out."

The net twist trapped in a ring alters the otherwise periodic boundary conditions by

$$
\begin{gathered}
\phi_{j+N}=\phi_{j}+2 \pi M_{\text {out }}, \\
\psi_{j+N}=\psi_{j}+2 \pi M_{\text {in }} .
\end{gathered}
$$

The governing equations are invariant to changes in $M_{\text {in }}$ and $M_{\text {out }}$ by $\pm N .^{21}$ We will assume that $M_{\mathrm{x}} \geqslant 0$, so that the number of excited pairs in a ring is simply $m_{\mathrm{a}, \mathrm{x}}$. Note that in our experiment $M_{\text {in }}$ can be different from $M_{\text {out }}$.

Due to the magnetic coupling between rings, the motion of kinks and antikinks in one ring excites linear waves in both rings. When $M_{\text {in }} \neq M_{\text {out }}\left(\right.$ and $\left.m_{\mathrm{a}, \text { in }}=m_{\mathrm{a}, \text { out }}=0\right)$, two linear waves are excited with wave numbers $\kappa_{1}=2 \pi M_{\text {out }} / N$ and $\kappa_{2}=2 \pi M_{\text {in }} / N$; however, the amplitudes of the linear waves in each ring may be different. The inductive coupling also causes splitting of the dispersion relation for these linear waves. When $M_{\text {in }}=M_{\text {out }}$, the calculation is the same as for an open-ended system, which, for $m$ kinks or antikinks $(\kappa=2 \pi m / N)$, gives an approximate dispersion relation ${ }^{32,27}$

$$
\omega^{ \pm}(m, \mathrm{x}) \approx \frac{2 \Lambda_{\mathrm{x}}|\sin (m \pi / N)|}{\sqrt{1-2 Q_{\mathrm{h}, \mathrm{x}} \cos (2 m \pi / N) \mp Q_{\mathrm{v}, \mathrm{x}}}} .
$$

This expression was derived for equal numbers of $m$ kinks or antikinks in coupled identical sine-Gordon systems, for small $\Gamma$. We will use Eq. (5) as a semiquantitative estimate in the more general cases. It should be noted that the dispersion (5) is considered to be valid when kinks trapped in a given ring are closely packed and overlap one another, i.e., $m \Lambda_{\mathrm{x}} / N \gg 1$. If they are well-separated and localized (i.e., $\left.m \Lambda_{\mathrm{x}} / N \ll 1\right)$, then one expects a correction term that becomes significant when $\Lambda_{x}$ becomes small. ${ }^{6,20}$ The difference in the dispersion relation depending on packing of the kinks has been recently studied more carefully by Zheng et $a l .^{33}$ as well as by Strunz and Elmer. ${ }^{34}$ 


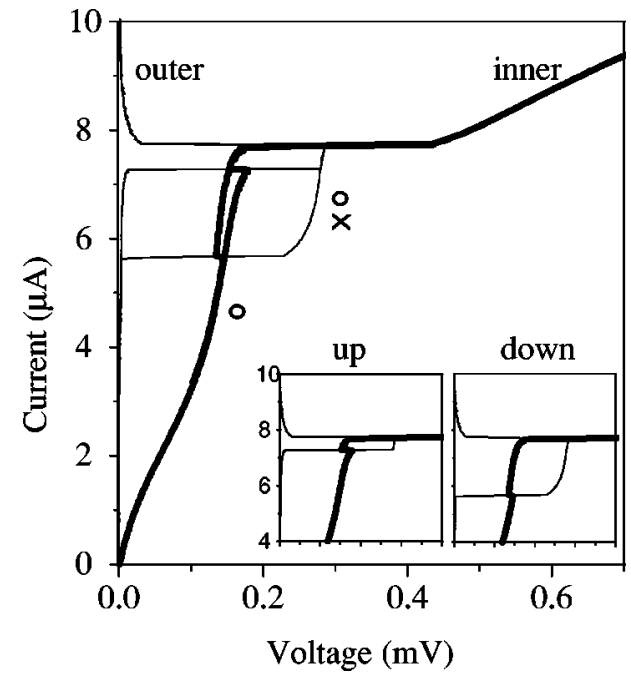

FIG. 2. Current-voltage curves for the $N=4$ system with $M_{\text {in }}$ $=1$ and $M_{\text {out }}=0$. The sample parameters are $L_{\text {in }}=21 \mathrm{pH}, L_{\text {out }}$ $=26 \mathrm{pH}$, and $j_{\mathrm{c}}(T=0)=139 \mathrm{~A} / \mathrm{cm}^{2} . T=7.22 \mathrm{~K}$, giving $\Gamma$ $=0.0823, \Lambda_{\text {in }}^{2}=2.55, \Lambda_{\text {out }}^{2}=2.07$.

\section{EXPERIMENTAL FEATURES OF THE SYSTEM}

We first choose a case where $M_{\text {in }}=1$ and $M_{\text {out }}=0$ in order to introduce some of the interesting features of our system. Figure 2 shows simultaneous measurements of the inner and outer ring voltages while the bias current is varied. In the figure, we use an open circle to represent kinks and a cross to represent the antikink. As the bias current is increased from zero, the single kink trapped in the inner ring begins to move, producing an average dc voltage across the inner ring junctions. Meanwhile, the outer ring, which has no trapped kinks, remains superconducting.

Since the system is discrete, the kink phase locks with linear waves in the system. ${ }^{20}$ As the kink's rotational speed $\omega / \kappa$ approaches the phase velocity for these linear waves, $\omega^{ \pm}(m, \mathrm{x}) / \kappa$, further increases in the bias current tend to increase the amplitudes of the excited linear waves rather than the speed of the kink. As a result, steps of almost constant voltage appear in the IV. When $m_{\mathrm{v}, \mathrm{x}}=M_{\mathrm{x}}$ so that there are no antivortices, we refer to the corresponding resonant step as a flux flow step (FFS). This is the discrete analog to the Eck step, ${ }^{35}$ which is also called a FFS, in continuous systems. ${ }^{36}$

As the inner ring approaches a constant voltage at the FFS, we can assume (and numerical simulations confirm) that the excited linear waves in both rings have significant amplitudes. When the current is monotonically increased from zero, at approximately $I_{\mathrm{b}}=7.2 \mu \mathrm{A}$ the outer ring's superconducting state becomes unstable, and a step appears. The up path of the IV is shown separately in the left inset of Fig. 2. This instability can be associated with the excitation of a kink/antikink pair. ${ }^{21,37}$ To lowest order, the contributions of the kinks and antikinks in a given ring simply add up, resulting in voltage steps at $\omega=\omega^{ \pm}\left(m_{\mathrm{v}}, \mathrm{x}\right)+\omega^{ \pm}\left(m_{\mathrm{a}}, \mathrm{x}\right) .{ }^{21}$ We refer to these as high-voltage steps (HVS), since they are similar in nature to the corresponding FFS but are higher in voltage.

As soon as the outer ring switches to the HVS, the voltage of the inner ring (on a FFS) shifts to a slightly lower value. The step which appears on the up path of the outer ring IV has approximately twice the voltage of the inner ring step. This is consistent with the picture that the outer ring kink and antikink move at nearly equal speeds and in opposite directions, producing twice the dc voltage of the kink moving in the inner ring. The sudden shift in the inner ring FFS voltage (which is clearer in the inset) indicates that the inner ring kink slows down when a pair appears in the outer ring. This suggests that the inner ring kink may have slowed to phase lock with the kink in the outer ring and that together they travel at a speed corresponding to $\omega^{-}$.

At still higher driving current, the excited pair disappears again as the FFS in the inner ring becomes unstable. At this point (near $7.75 \mu \mathrm{A}$ ), the outer ring switches back to the superconducting state and the inner ring switches to a uniform whirling state. ${ }^{7}$ On the down path (the right inset of Fig. 2 with current decreasing monotonically from $10 \mu \mathrm{A}$ ), a pair is again excited in the outer ring and persists as the current bias is decreased even below $7.2 \mu \mathrm{A}$, causing hysteresis in the IV. Surprisingly, the voltage of this HVS gradually decreases to well below twice the voltage of the inner ring FFS. When the pair disappears from the outer ring, the inner ring kink speeds up again, as indicated by the shift in the FFS voltage at $I=5.6 \mu \mathrm{A}$.

The experiments show two surprising results. First, a shift to a lower voltage occurs in the FFS as soon as the outer ring is on the HVS. This adjustment of the kink velocity clearly illustrates the existence of interactions between the two rings. Second, the voltage of the FFS is not exactly half of the voltage of the HVS. This observation indicates that not the whole array is phase-locked. We have performed many simulations to study these aspects in more detail. Some results will be presented in the next section.

\section{SIMULATIONS: KINK AND ANTIKINK INTERACTIONS}

In order to better understand the interactions described by the data in Fig. 2, we have simulated the system with parameters similar to our experiments. However, we find that simulations of systems with larger $N$, where the kinks are very localized and easy to identify, are the most elucidating. Figure 3 shows an IV characteristic from a simulation. In this case, there are $N=51$ junctions in each ring. For a range of bias currents $\left(I_{b}<0.57 I_{c}\right)$, kink/antikink pairs are excited in the outer ring. The inset shows the relative motions of the kinks and the antikink when one pair is excited in the outer ring.

In Fig. 3, we plot the average dc voltage for each ring vs bias current. As the current is increased, the kinks move faster, and the dc voltage increases. The inner ring FFS reaches a maximum at a voltage of $0.0151 I_{c} R_{n}$. The outer ring has a phase-winding of zero, but exhibits HVS. The first step in the outer ring at $0.0297 I_{\mathrm{c}} R_{\mathrm{n}}$ is due to a single excited pair. As in the experiment, the voltage of the FFS step jumps to a lower voltage value when the outer ring switches to the HVS.

Along the FFS, we find that the average voltage of the outer ring is not quite twice that of the inner ring's, indicating that the speeds of the two kinks and the antikink are not identical. Figure 4 plots the space-time portrait of the motion at $I_{b}=0.45 I_{\mathrm{c}}$. The two kinks, drawn as open circles, travel 


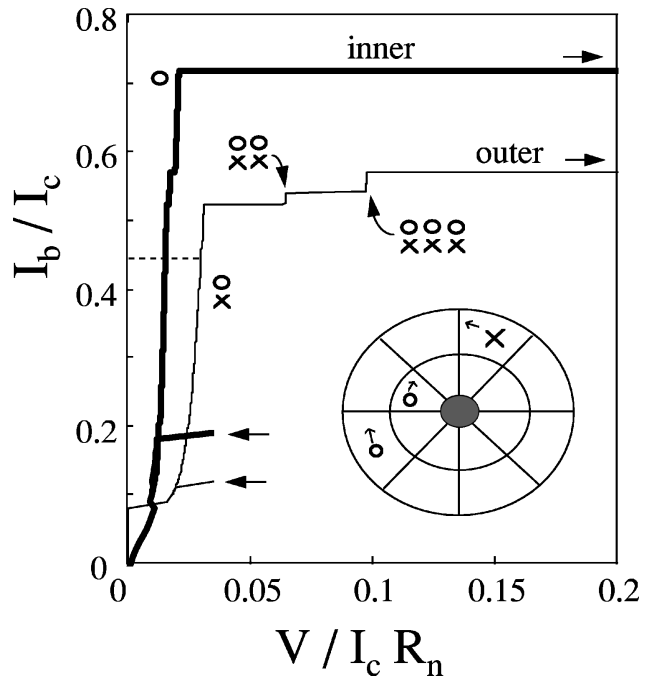

FIG. 3. Simulated IV characteristic for parameters: $M_{\text {in }}=1$, $M_{\text {out }}=0, \quad N=51, \quad \Gamma=0.1, \quad \Lambda_{\text {out }}^{2}=1.76, \quad \Lambda_{\text {in }}^{2}=2.55, \quad Q_{\mathrm{h}, \text { out }}=0.08$, $Q_{\mathrm{v}, \text { out }}=0.12, Q_{\mathrm{h}, \text { in }}=0.12$, and $Q_{\mathrm{v}, \text { in }}=0.17$. The nearly constant voltage steps correspond to the motion of the kinks (represented by open circles) and antikinks (represented by crosses) at their maximum speeds. The inset shows the relative motion of the kinks and the antikink for one excited pair in the outer ring.

at approximately the same speed with a constant phase separation between them. Hence, we see that the coupling gives rise to phase-locking between kinks of the same sign in different rings.

A more dramatic interaction effect is the precession of the collision region between the kink and antikink in the outer ring. The antikink travels in the opposite direction and moves more slowly than the kink. As it periodically collides with the kink, the interaction perturbs its motion slightly. Because of the difference in their speeds, they collide at a different location for each revolution, and the collision region precesses around the ring, as shown from the solid line

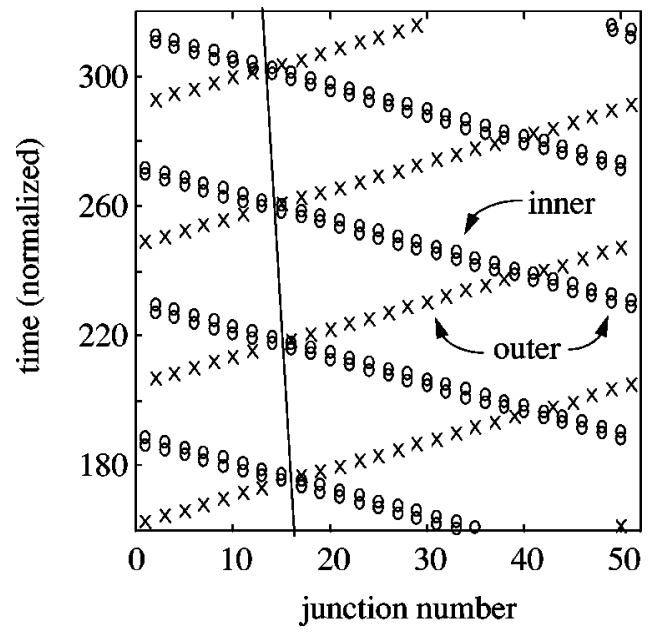

FIG. 4. Space-time diagram of the kinks for $I_{b}=0.45 I_{\mathrm{c}}$ marked by a dashed line in Fig. 2. The kinks are represented by open circles, while the antikink is marked by crosses. The kinks in the two rings are phase locked (note the parallel lines of circles). The antikink travels more slowly, causing its point of intersection with the kink to precess.

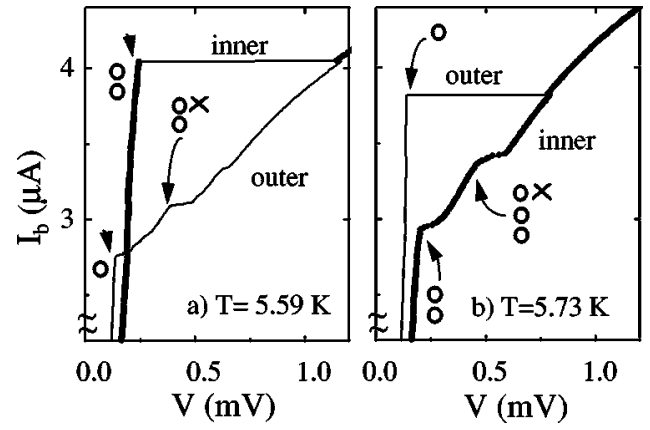

FIG. 5. Measured IV characteristic with $N=4$. These close-ups begin at a bias current of $2.2 \mu \mathrm{A}$, when both rings are on FFS. The sample parameters are $L_{\text {out }}=26 \mathrm{pH}, L_{\text {in }}=21 \mathrm{pH}$, and $j_{\mathrm{c}}(T=0)$ $=83.3 \mathrm{~A} / \mathrm{cm}^{2} . M_{\text {in }}=2$ and $M_{\text {out }}=1$. (a) $T=5.59 \mathrm{~K}$, giving $\Gamma$ $=0.0507, \Lambda_{\text {in }}^{2}=2.67$, and $\Lambda_{\text {out }}^{2}=2.23$. A kink/antikink pair is excited in the outer ring at $I_{\mathrm{b}}=2.75 \mu \mathrm{A}$, causing an HVS. (b) $T$ $=5.73 \mathrm{~K}$, giving $\Gamma=0.0514, \Lambda_{\text {in }}^{2}=2.74$, and $\Lambda_{\text {out }}^{2}=2.29$. A kink/ antikink pair is excited in the inner ring at $I_{\mathrm{b}}=2.9 \mu \mathrm{A}$.

in Fig. 4. Slopes of the lines indicate that $\omega\left(m_{\mathrm{v}, \text { in }}\right)$ $=\omega\left(m_{\mathrm{v}, \text { out }}\right)=0.0151$, and $\omega\left(m_{\mathrm{a}, \text { out }}\right)=0.0146$. The difference $\omega\left(m_{\mathrm{v}, \text { out }}\right)-\omega\left(m_{\mathrm{a}, \text { out }}\right)$ gives the precession rate of the kink/ antikink collision region. This difference may be attributed to the two kinks traveling at a higher speed which approaches $\omega^{+}(1$,out $)$ in Eq. (5) while the speed of the antikink approaches $\omega^{-}(1$, out $){ }^{38}$

When the current is increased above $I_{\mathrm{b}}=0.52 I_{\mathrm{c}}$, a second pair is excited in the outer ring and a voltage step appears at $0.065 I_{\mathrm{c}} R_{\mathrm{n}}$. At this point, the inner ring voltage shifts to a slightly higher value. Since the system is discrete, we might expect the two vortices in the outer ring to travel more slowly than the single vortex in the inner ring. Instead, space-time plots (not shown) indicate that, again, the kinks in both rings move together at equal speeds and the antikinks in the outer ring move together at a slower speed. When a third pair is excited at $I_{\mathrm{b}}=0.54 I_{\mathrm{c}}$, the FFS voltage of the inner ring does not change. Finally, at $I_{\mathrm{b}}=0.57 I_{\mathrm{c}}$ the pairs disappear and the outer ring switches to a uniform whirling state. ${ }^{7}$ The inner branch persists up to higher current and jumps at $I_{\mathrm{b}}=0.72 I_{\mathrm{c}}$.

\section{EXPERIMENTS: A SYSTEM WITH MANY STATES}

Now we study the system when there are nonzero phase windings in both of the rings $\left(M_{\mathrm{x}} \neq 0\right)$. To illustrate, $M_{\text {in }}$ $=2$ and $M_{\text {out }}=1$ are used in Fig. 5, which shows a close-up of the FFS in both rings above $I_{\mathrm{b}}=2.2 \mu \mathrm{A}$. There are two kinks (drawn as open circles) trapped in the inner ring and one in the outer ring. As the current bias is increased along the FFS, the amplitudes of excited linear waves grow until at least one of the FFS destabilizes. At this point, one or both rings may switch to a HVS with the excitation of one or more kink/antikink pairs.

In Fig. 5(a) the HVS occurs at $I_{\mathrm{b}}=2.75 \mu \mathrm{A}$, when a kink/ antikink pair is excited in the outer ring, causing the voltage across the outer ring to increase. The IV steepens as the two kinks and the single antikink reach their maximum speeds. In both experiments and simulations, we observe additional steps at voltages corresponding to: $\omega=n_{v} \omega^{ \pm}\left(m_{\mathrm{v}}, \mathrm{x}\right)$ $+n_{a} \omega^{ \pm}\left(m_{\mathrm{a}}, \mathrm{x}\right)$, where $n_{v}$ and $n_{a}$ are integers. Although 
there are many possible combinations of FFS and HVS states for the system of two rings, we observe only a limited set of states. In fact, for the case $n_{a}=n_{v}=1$, our measurements of $N=4$ and $N=8$ systems exhibit only one out of the possible HVS for a given $M_{\text {in }}$ and $M_{\text {out }}$.

Even more intriguing is the fact that, unless $M_{\text {in }}=M_{\text {out }}$, HVS appear in only one ring at a time. An interesting question, then, is which ring will have an excited pair and when. The answer depends on temperature, which directly affects the parameters $\Lambda_{\mathrm{x}}$ and $\Gamma$. Figure 5(b) shows the IV at a slightly higher temperature, corresponding to an increase of $\Lambda_{\mathrm{x}}$ and $\Gamma$ by only 1.3 percent from the IV in Fig. 5(a). At this temperature an HVS appears in the inner ring instead.

We find that for every distinct combination of $M_{\text {in }}$ $\neq M_{\text {out }}$, a parameter regime where pairs are excited only in the outer ring, another parameter regime where pairs are excited only in the inner ring, and a transition region in temperature from one state to the other. The reason for a transition from one state to another as the parameters are varied is related to the stability of the states. In fact, we observe similar transitions among many of the possible dynamical states of the system. For a given $M_{\text {in }}$ and $M_{\text {out }}$, each ring can be in one of the following states: (1) $V=0$, (2) FFS, (3) HVS, and (4) whirling branch (admittedly a simplified picture, for the purpose of discussion), leading to 16 possibilities for the system. Which of these possible states appears depends on both the driving current and the system parameters. As seen in Fig. 2, more than one state can be stable for a given driving current when the system is hysteretic.

We find a surprising feature in the evolution of the coupled ring IV curves as the temperature is varied. As the temperature is monotonically changed, the range of bias currents for which a given state is stable can shrink to almost zero until a new state appears at that current value. At this transition temperature, there is no visible hysteresis in the IV curves. This situation is illustrated in Fig. 2. At about $I_{\mathrm{b}}$ $=7.75 \mu \mathrm{A}$, the system switches from a combination of [in $=$ FFS, out $=$ HVS $]$ to $[$ in $=$ whirling branch, out $=$ zero voltage], with no hysteresis. While this may turn out to be a simple coincidence, the result can be dramatic experimentally. The tiniest bit of AC drive added to the input current can switch the system between two largely different voltage outputs. In the future, these transitions will be studied in more detail.

\section{SUMMARY}

We have studied the dynamics of two inductively coupled Josephson rings. Different numbers of kinks (vortices) can be trapped in each of the rings. A generic feature of our data is that voltage shifts in one ring occur when the other ring changes its dynamic state such that kinks and antikinks are excited or annihilated. Numerical simulations show that a phase locking occurs between the kinks in separate rings but the antikinks may move at different velocities. The two rings are therefore not completely phase-locked and a precession of the kink/antikink collision region occurs.

The dynamics is especially complicated when each ring contains kinks but not of the same number. Our experiments show that kinks and antikinks are only excited in one ring at the time. In which ring these pairs are excited depends on the system parameters (i.e., the temperature in the experiment). Many details of the interaction effects between the two coupled rings are not understood at present. For instance, we cannot quantitatively explain the voltage shifts that occur when kinks and antikinks are excited in the other ring. Nor do we have a complete picture of the intriguing transitions between dynamical states which occur at a single point in parameter space and are characterized by a sudden absence of hysteresis in the IV curve. The present paper only presents an exploration of the interesting interaction effects between kinks in two coupled rings of nonlinear oscillators. As such, a system of two coupled Josephson rings is an ideal model for further studies.

\section{ACKNOWLEDGMENTS}

The research was supported in part by the NSF Graduate Fellowship program and NSF Grants DMR-9610042 and DMS-9500948. We thank L. Mahadevan for useful discussions. We are especially grateful to Shinya Watanabe for his critical review of the manuscript.
${ }^{1}$ A. M. Turing, Philos. Trans. R. Soc. London, Ser. B 237, 37 (1953).

${ }^{2}$ G. Goldsztein and S. H. Strogatz, Int. J. Bifurcation Chaos Appl. Sci. Eng. 5, 983 (1995).

${ }^{3}$ M. de Sousa Vieira, A. J. Lichtenberg, and M. A. Lieberman, Int. J. Bifurcation Chaos Appl. Sci. Eng. 4, 1563 (1994).

${ }^{4}$ V. S. Afraimovich, V. I. Nekorkin, G. V. Osipov, and V. D. Shalfeev, Stability, Structures and Chaos in Nonlinear Synchronization Networks (World Scientific, Singapore, 1994).

${ }^{5}$ M. Silber, L. Fabiny, and K. Wiesenfeld, J. Opt. Soc. Am. B 10, 1121 (1993).

${ }^{6}$ A. V. Ustinov, M. Cirillo, and B. A. Malomed, Phys. Rev. B 47, 8357 (1993).

${ }^{7}$ S. Watanabe, H. S. J. van der Zant, S. H. Strogatz, and T. P. Orlando, Physica D 97, 429 (1996).

${ }^{8}$ M. Levi, in Analysis, Et Cetera, edited by P. Rabinowitz and E.
Zehnder (Academic Press, New York, 1990), p. 471.

${ }^{9}$ I. R. Epstein and M. Golubitsky, Chaos 3, 1 (1993).

${ }^{10}$ J. F. Heagy, T. L. Carroll, and L. M. Pecora, Phys. Rev. Lett. 73, 3528 (1994); J. F. Heagy, L. M. Pecora, and T. L. Carroll, ibid. 74, 4185 (1995).

${ }^{11}$ A. T. Winfree, The Geometry of Biological Time (Springer, New York, 1980), p. 127.

${ }^{12}$ G. B. Ermentrout, J. Math. Biol. 23, 55 (1985); G. B. Ermentrout and J. Rinzel, ibid. 11, 269 (1981).

${ }^{13}$ A. Davidson, B. Dueholm, B. Kryger, and N. F. Pedersen, Phys. Rev. Lett. 55, 2059 (1985).

${ }^{14}$ K. Maginu, SIAM (Soc. Ind. Appl. Math.) J. Appl. Math. 43, 225 (1983).

${ }^{15}$ A. V. Ustinov, T. Dorderer, R. P. Huebener, N. F. Pedersen, B. Mayer, and V. A. Oboznov, Phys. Rev. Lett. 69, 1815 (1992).

${ }^{16}$ I. V. Vernik, N. Lazarides, M. P. Sorensen, A. V. Ustinov, N. F. 
Pedersen, and V. A. Oboznov, J. Appl. Phys. 79, 7854 (1996).

${ }^{17}$ N. Martucciello, J. Mygind, V. P. Koshelets, A. V. Shchukin, L. V. Filippenko, and R. Monaco, Phys. Rev. B 57, 5444 (1998).

${ }^{18}$ J. F. Currie, S. E. Trullinger, A. R. Bishop, and J. A. Krumhansl, Phys. Rev. B 15, 5567 (1977).

${ }^{19}$ M. Peyrard and M. D. Kruskal, Physica D 14, 88 (1984).

${ }^{20}$ H. S. J. van der Zant, T. P. Orlando, S. Watanabe, and S. H. Strogatz, Phys. Rev. Lett. 74, 174 (1995).

${ }^{21}$ S. Watanabe, S. H. Strogatz, H. S. J. van der Zant, and T. P. Orlando, Phys. Rev. Lett. 74, 379 (1995).

${ }^{22}$ P. Barbara, A. V. Ustinov, and G. Costabile, Phys. Lett. A 191, 443 (1994).

${ }^{23}$ E. Goldobin, A. Wallraff, N. Thyssen, and A. V. Ustinov, Phys. Rev. B 57, 130 (1998).

${ }^{24}$ A. Petraglia, A. V. Ustinov, N. F. Pedersen, and S. Sakai, J. Appl. Phys. 77, 1171 (1995).

${ }^{25}$ A. Wallraff, E. Goldobin, and A. V. Ustinov, J. Appl. Phys. 80, 6523 (1996).

${ }^{26}$ S. Sakai, P. Bodin, and N. F. Pedersen, J. Appl. Phys. 73, 2411 (1993)

${ }^{27}$ A. Petraglia, N. F. Pedersen, P. L. Christiansen, and A. V. Ustinov, Phys. Rev. B 55, 8490 (1997).

${ }^{28}$ R. D. Parmentier, P. Barbara, G. Costabile, A. D'Anna, B. A.
Malomed, and C. Soriano, Phys. Rev. B 55, 15165 (1997).

${ }^{29}$ N. Flytzanis, S. Crowley, and V. Celli, Phys. Rev. Lett. 39, 891 (1977).

${ }^{30}$ A. R. Bishop and T. F. Lewis, J. Phys. C 12, 3811 (1979).

${ }^{31}$ Samples were fabricated by HYPRES, Inc. in Elmsford, NY.

${ }^{32}$ A. E. Duwel, E. Trías, T. P. Orlando, H. S. J. van der Zant, S. Watanabe, and S. H. Strogatz, J. Appl. Phys. 79, 7864 (1996).

${ }^{33}$ Zhigang Zheng, Bambi $\mathrm{Hu}$, and Gang $\mathrm{Hu}$, cond-mat/9802251 (unpublished).

${ }^{34}$ T. Strunz and F. Elmer, cond-mat/9805287 (unpublished).

${ }^{35}$ R. E. Eck, D. J. Scalapino, and B. N. Taylor, Phys. Rev. Lett. 13, 15 (1964).

${ }^{36}$ N. F. Pedersen and A. V. Ustinov, Supercond. Sci. Technol. 8, 389 (1995).

${ }^{37}$ G. Costabile, S. Pagano, and R. D. Parmentier, Phys. Rev. B 36, 5225 (1987).

${ }^{38}$ In smaller systems where the kink or antikink spacing becomes of the order of the ring size, this interaction-induced precession is more difficult to identify. However, the excited linear waves clearly show the presence of both frequencies, even when the rings are identical, and a beat frequency, $\omega^{+}\left(m_{\mathrm{v}}\right)-\omega^{-}\left(m_{\mathrm{a}}\right)$, appears. 